\title{
Replication forks, chromatin loops and dormant replication origins $\mathrm{J}$ Julian Blow and Xin Quan Ge
}

Address: Wellcome Trust Centre for Gene Regulation and Expression, College of Life Sciences, University of Dundee, Dow Street, Dundee DD1 5EH, UK.

Correspondence: J Julian Blow. Email: j.j.blow@dundee.ac.uk

Published: 30 December 2008

Genome Biology 2008, 9:244 (doi:10.1 186/gb-2008-9-12-244)

The electronic version of this article is the complete one and can be found online at http://genomebiology.com/2008/9//2/244

(c) 2008 BioMed Central Ltd

\begin{abstract}
When DNA replication is slowed down, normally dormant replication origins are activated. Recent work demonstrates that cells adapt by changing the organization of chromatin loops and maintaining the new pattern of origin use in subsequent cell cycles.
\end{abstract}

It is critical that chromosomal DNA is precisely duplicated during $\mathrm{S}$ phase of the eukaryotic cell cycle, with no sections of DNA left unreplicated or replicated more than once. There is a considerable plasticity in this process because cells license many potential replication origins, of which only a small percentage are used in any one cell cycle, with the others remaining 'dormant'. This means that the usage of replication origins can change under different circumstances. For example, dormant replication origins can be activated when replication forks are inhibited to allow timely completion of the replication programme. A recent paper published in Nature by Courbet et al. [1] illustrates this plasticity of replication origin usage and shows that it is associated with longer-term changes to the organization of chromatin loops. The changes to chromatin organization can then directly affect the way that replication origins are used in subsequent cell cycles.

\section{Dormant origins and the plasticity of the replication program}

The precise duplication of large eukaryotic chromosomes is a dauntingly complex task. For the DNA to be completely replicated, replication forks need to be initiated at thousands of replication origins scattered throughout the genome. This is made more difficult by the fact that replication forks can frequently stall, for example if they encounter damaged bases. It is also crucial that each replication origin does not fire more than once in a single $\mathrm{S}$ phase, as this would lead to local amplification of the DNA.
During late mitosis and early $\mathrm{G} 1$, the cell licenses replication origins for use in the upcoming $\mathrm{S}$ phase by loading protein complexes composed of Mcm proteins (Mcm2-7 complexes) onto the origin DNA $[2,3]$. During S phase, Mcm2-7 at licensed origins can initiate replication forks. The Mcm2-7 complex moves with the replication forks, providing the essential DNA helicase activity that unwinds the DNA. This means that when an origin initiates a pair of forks, it is converted into the unlicensed state and cannot fire again. However, the mechanisms that ensure the appropriate distribution and usage of replication origins on DNA are poorly understood in animal cells. Many more origins are licensed in G1 than are actually used, with around 90\% of licensed origins being inefficient and remaining dormant in any given $\mathrm{S}$ phase. When replication forks are stalled or slowed, dormant origins are activated [4-6], which can help cells ensure complete genome replication [7-9].

Activation of dormant origins by a 'passive' mechanism In their recent paper, Courbet et al. [1] investigated the regulation of origin usage in response to changes in replication fork dynamics. Previous work from their lab had mapped a cluster of replication origins in an amplified region surrounding the AMPD2 (adenosine monophosphate deaminase 2) locus in Chinese hamster fibroblasts [10]. Under conditions of normal fork movement, this region is predominantly replicated from forks initiated at an origin termed oriGNAI3, though initiation was occasionally observed at inefficient (dormant) origins termed oriA-oriF. When forks were slowed, by 
(a) Cells grown in medium that allows only slow fork movement
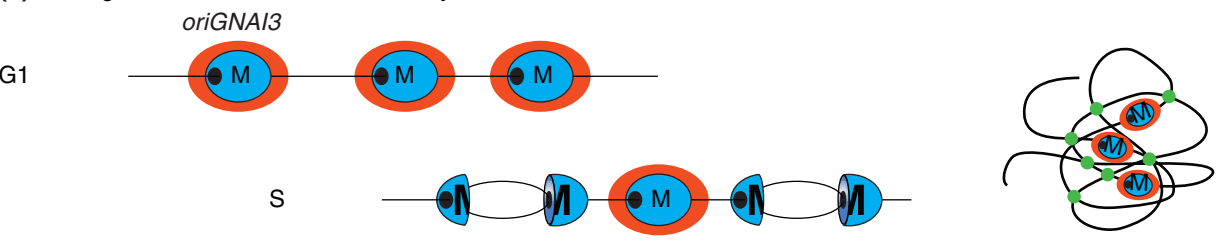

(b) Switch to medium that promotes fast fork movement

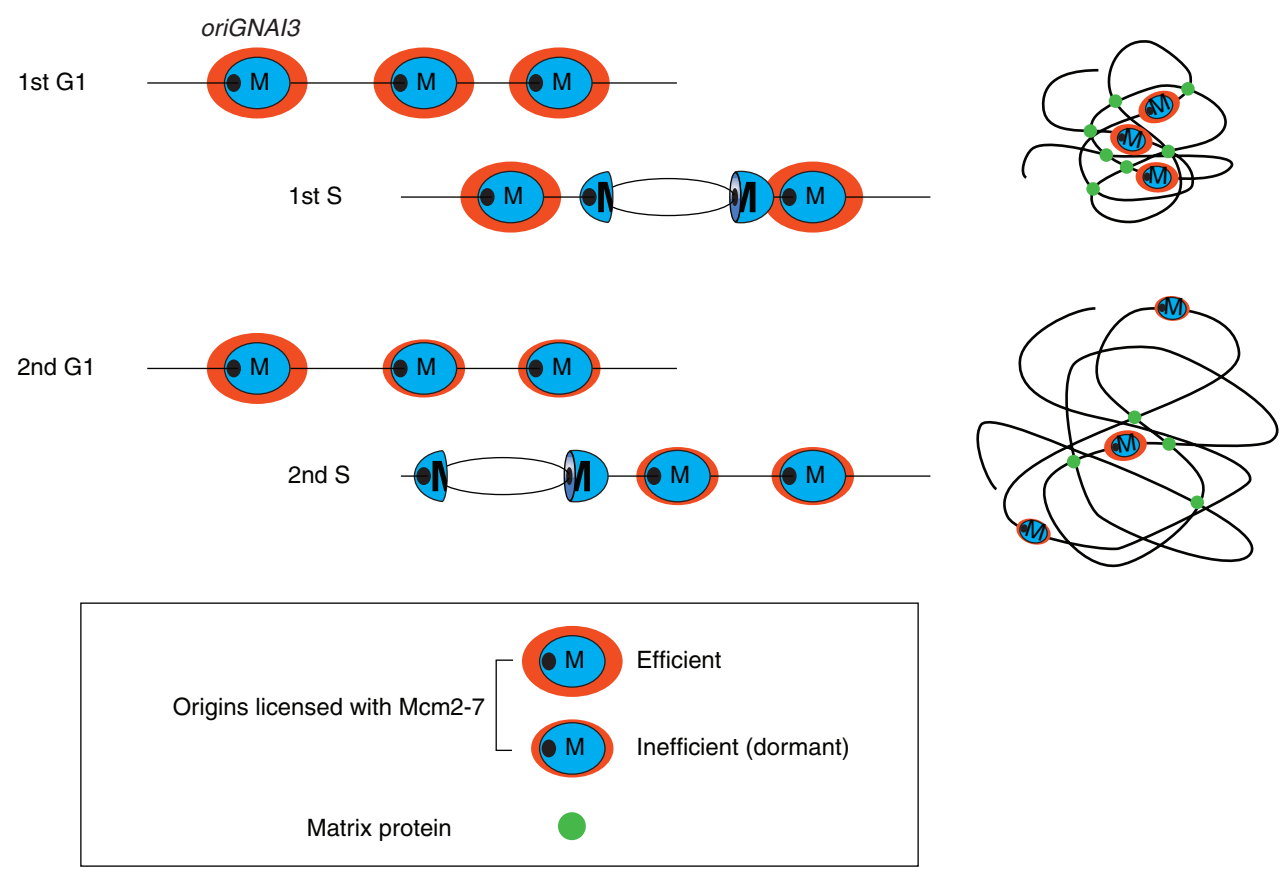

Figure I

A simplified version of the AMPD2 locus is shown, with the primary origin oriGNA/3 on the left and two less efficient origins on the right. During GI, origins are licensed by binding Mcm2-7 (blue, M); when origins fire during S phase, Mcm2-7 provides essential helicase activity at the fork. The cartoons on the right show the chromatin of the locus coiled up and cross-linked to proteins of the nuclear matrix (green dots), forming a 'halo' of DNA around the tethering points. (a) Cells adapted for growth under conditions of slow fork movement. Multiple origins fire in the locus, with all origins having become relatively efficient (large red ovals) to compensate for slow fork movement, and all being associated with matrix proteins. (b) In the first cell cycle after a shift to conditions allowing fast fork progression, the rate of origin firing is decreased, but the relative efficiency and the matrix-association properties of the origins are similar to those seen before the shift. Only in the second cell cycle after the shift do the two relatively inefficient origins become dormant again (small red ovals) and less closely associated with the matrix.

reducing the cellular supply of deoxynucleotides, initiation at oriA-oriF was significantly increased. This was associated with an overall increase in the number of initiation events throughout the locus.

One simple explanation for these results is that origin firing is stochastic. Once a genomic locus containing a cluster of origins becomes activated, dormant origins within that cluster normally have only a brief time to fire before they are passively replicated (and hence inactivated) by a fork from a neighboring origin. If replication forks are slowed, dormant origins are more likely to fire simply because there is an increased period of time before they are passively replicated. Consistent with this 'passive' mechanism, it has been reported that under conditions of modest fork slowing (when replication checkpoints are not strongly activated), there is a correlation between the degree of fork slowing and the overall increase in origin density $[1,8]$.

\section{Long-term adaptation of origin usage}

This passive activation of dormant origins is a rapid and transitory response that should not affect the long-term behavior of cells. A key observation of Courbet et al. [1] is 
that in addition to this rapid response, cells also respond to changes in fork dynamics by adapting origin usage in subsequent cell cycles. Cells were grown under conditions in which forks could only move slowly, promoting a high rate of initiation at oriA-oriF as well as at oriGNAI3 (Figure 1). Cells were then synchronized in mitosis and replated into fresh medium that allows fast fork progression. In the first $S$ phase after replating, the overall initiation density in the $A M P D 2$ locus dropped, so that there was a low frequency of multiple initiations occurring in individual loci, as expected by the 'passive' mechanism. However, initiation did not occur predominantly at the primary origin, oriGNAI3, but was distributed among the dormant origins oriA-oriF as well as oriGNAI3. Only in the second S phase after the increase in fork speed was the dominance of oriGNAI3 regained and the relative efficiency of initiation at oriA-oriF decreased. It therefore appears that cells had adapted to growth under slow fork conditions by raising the efficiency of oriA-oriF, which under normal conditions usually remain dormant.

\section{Changes to chromatin loops correlate with the adaptation}

Courbet et al. [1] go on to show that the adaptation of origin efficiency correlates both with changes in chromatin organization and the association of replication origins with the nuclear matrix in G1. Previous work has shown that during $\mathrm{S}$ phase, clusters of 5-50 adjacent replicons (the stretch of DNA replicated from a given origin) are replicated together in 'factories', with all the DNA replicated in a single factory remaining co-localized within the nucleus over many cell cycles [11-13]. The physical basis for this organization is currently unknown. One suggestion is that it reflects the attachment of specific DNA sequences to an insoluble nucleoskeleton or matrix, thereby creating chromatin loops that define functional units of transcription and replication [14]. A slightly different view is that chromatin loops may be held together by multiple weak and reversible interactions between chromatin-bound proteins $[15,16]$. Whatever its physical basis, there is known to be a good correlation between the size of DNA loops in the 'halo' of DNA that appears to be tethered to the nuclear matrix and the average spacing between replication origins [17].

When Courbet et al. grew cells for several generations under conditions where replication forks moved slowly, both the primary (oriGNAI3) and dormant (oriA-oriF) origins were used with similar efficiencies, and they were distributed fairly similarly throughout the halo of matrix-attached DNA. The total size of these halos was smaller than that of halos from cells grown under normal conditions (see cartoons on the right-hand side of Figure 1). When cells were grown for several generations under conditions that allow fast fork progression, the DNA halos became larger (consistent with the lower average density of origins [17]), and oriGNAI3, but not the dormant origins, was preferentially found closer to the center of the halo. Critically, when synchronized cells were changed from 'slow-fork' conditions to conditions allowing normal fork rates, the change in halo size and the relative positions of the origins within the halo were only seen in the second cell cycle after the switch (Figure 1b).

This suggests that the positioning of origins within the halo, which correlates with the relative efficiency of these origins, is a long-term adaptation to changes in fork rate that persists into the next cell cycle. In some way, information about how the origins in a cluster have been replicated is converted into a changed organization of the nuclear matrix and the attachment of replication origins to it. Because we do not understand how replication origins are organized within replication factories and in chromatin loops, we can only speculate on what these changes might be. Some marker of where forks initiate or terminate might be left on the DNA. Proteins such as topoisomerase II [18-20] and cohesin $[21,22]$, which are involved in the organization of DNA within the nucleus and which interact with the replication machinery, could be involved. When such proteins are deposited on chromatin, this might physically bring origins together with the matrix to increase their firing efficiency.

The Mcm2-7 proteins that license replication origins are commonly misregulated at an early stage in cancer cells [23-25], and the incorrect regulation of replication origins may be an important cause of the genetic instability seen in cancer. The work of Courbet et al. [1] confirms the plasticity of origin usage during DNA replication and provides clues as to how origin selection might occur in animal cells. Although this problem has been apparent for many years, there is now promise that it can be better understood.

\section{References}

I. Courbet S, Gay S, Arnoult N, Wronka G, Anglana M, Brison O, Debatisse M: Replication fork movement sets chromatin loop size and origin choice in mammalian cells. Nature 2008, 455:557-560.

2. Blow JJ, Dutta A: Preventing re-replication of chromosomal DNA. Nat Rev Mol Cell Biol 2005, 6:476-486.

3. Arias EE, Walter JC: Strength in numbers: preventing rereplication via multiple mechanisms in eukaryotic cells. Genes Dev 2007, 21:497-5 I8.

4. Ockey $\mathrm{CH}$, Saffhill R: The comparative effects of short-term DNA inhibition on replicon synthesis in mammalian cells. Exp Cell Res 1976, 103:36|-373.

5. Taylor $\mathrm{JH}$ : Increase in DNA replication sites in cells held at the beginning of S phase. Chromosoma 1977, 62:291-300.

6. Gilbert DM: Replication origin plasticity, Taylor-made: inhibition vs recruitment of origins under conditions of replication stress. Chromosoma 2007, I 16:341-347.

7. Woodward AM, Gohler T, Luciani MG, Oehlmann M, Ge X, Gartner A, Jackson DA, Blow Jj: Excess Mcm2-7 license dormant origins of replication that can be used under conditions of replicative stress. J Cell Biol 2006, 173:673-683.

8. Ge XQ, Jackson DA, Blow Jj: Dormant origins licensed by excess Mcm2-7 are required for human cells to survive replicative stress. Genes Dev 2007, 21:333 I-334I.

9. Ibarra A, Schwob E, Mendez J: Excess MCM proteins protect human cells from replicative stress by licensing backup origins of replication. Proc Natl Acad Sci USA 2008, 105:8956-8961. 
10. Anglana M, Apiou F, Bensimon A, Debatisse M: Dynamics of DNA replication in mammalian somatic cells: nucleotide pool modulates origin choice and interorigin spacing. Cell 2003, I 14:385-394.

II. Ferreira J, Paolella G, Ramos C, Lamond Al: Spatial organization of large-scale chromatin domains in the nucleus: a magnified view of single chromosome territories. J Cell Biol 1997, 139:1597-1610.

12. Jackson DA, Pombo A: Replicon clusters are stable units of chromosome structure: evidence that nuclear organization contributes to the efficient activation and propagation of $S$ phase in human cells. $J$ Cell Biol 1998, I40:1285-1295.

13. Berezney R, Dubey DD, Huberman JA: Heterogeneity of eukaryotic replicons, replicon clusters, and replication foci. Chromosoma 2000, 108:47I-484.

14. Jackson DA: The principles of nuclear structure. Chromosome Res 2003, II:387-40I.

15. Marko JF: Micromechanical studies of mitotic chromosomes. Chromosome Res 2008, 16:469-497.

16. Tsutsui KM, Sano K, Tsutsui K: Dynamic view of the nuclear matrix. Acta Med Okayama 2005, 59:1 I3-120.

17. Buongiorno-Nardelli M, Micheli G, Carri MT, Marilley M: A relationship between replicon size and supercoiled loop domains in the eukaryotic genome. Nature 1982, 298:100-102.

18. Abdurashidova G, Radulescu S, Sandoval O, Zahariev S, Danailov MB, Demidovich A, Santamaria L, Biamonti G, Riva S, Falaschi A: Functional interactions of DNA topoisomerases with a human replication origin. $E M B O$ J 2007, 26:998-1009.

19. Bermejo R, Doksani Y, Capra T, Katou YM, Tanaka H, Shirahige K, Foiani M: Topl- and Top2-mediated topological transitions at replication forks ensure fork progression and stability and prevent DNA damage checkpoint activation. Genes Dev 2007, 21:1921-1936.

20. Lemaitre JM, Danis E, Pasero P, Vassetzky Y, Mechali M: Mitotic remodeling of the replicon and chromosome structure. Cell 2005, |23:787-80|.

21. Parelho V, Hadjur S, Spivakov M, Leleu M, Sauer S, Gregson HC Jarmuz A, Canzonetta C, Webster Z, Nesterova T, Cobb BS, Yokomori K, Dillon N, Aragon L, Fisher AG, Merkenschlager M: Cohesins functionally associate with CTCF on mammalian chromosome arms. Cell 2008, 132:422-433.

22. West AG, Fraser P: Remote control of gene transcription. Hum Mol Genet 2005, I4 Spec No I:R IOI-RIII.

23. Blow JJ, Gillespie PJ: Replication licensing and cancer - a fatal entanglement? Nat Rev Cancer 2008, 8:799-806.

24. Gonzalez MA, Tachibana KE, Laskey RA, Coleman N: Control of DNA replication and its potential clinical exploitation. Nat Rev Cancer 2005, 5:|35-|4|.

25. Williams GH, Stoeber K: Cell cycle markers in clinical oncology. Curr Opin Cell Biol 2007, 19:672-679. 